RESEARCH REPORT

\title{
Variations in health status within and between socioeconomic strata
}

\author{
R L Ferrer, R Palmer
}

J Epidemiol Community Health 2004;58:381-387. doi: 10.1136/jech.2002.003251

See end of article for authors' affiliations

.....................

Correspondence to:

Dr R L Ferrer, Department

of Family and Community

Medicine University of

Texas Health Science

Center at San Antonio,

7703 Floyd Curl Drive,

San Antonio, TX

78229-3900, USA

ferrerr@uthscsa.edu

Accepted for publication 4 July 2003

\begin{abstract}
Objectives: To analyse the variability in health status within as well as between socioeconomic groups. What is the range of individual variability in the health effects of socioeconomic status? Is the adverse effect of lower socioeconomic status uniform across the entire distribution of health status?

Design: Nationally representative telephone survey of the US population in 1996.

Setting: 60 US metropolitan and rural areas.

Participants: 47076 adult respondents to the community tracking study.

Main outcome measures: Self rated physical and mental health status, measured by the Short Form-12 instrument.

Results: There is considerable variability in self rated health within socioeconomic strata and that variability increases in a step-wise fashion at each lower stratum of income. Most of the increased variability is accounted for by changes in the middle and lower (10th, 25th, and 50th centiles) rather than the upper (75th and 90th) portions of the distribution. A resilient subgroup of lower socioeconomic status people seems to maintain excellent self rated health throughout life, while a more vulnerable lower socioeconomic status group experiences rapid deterioration in health status as people reach middle age. Conclusions: Within the population level social structuring of health there are differences in individual resilience and vulnerability that are amenable to further exploration and potential modification.
\end{abstract}

$\mathrm{T}$ here is an inverse gradient between socioeconomic status (SES) and health across the spectrum of health conditions, including diseases, ${ }^{1}$ risk factors, ${ }^{1}$ emotional distress $^{2{ }^{3}}$ functional status, ${ }^{4}$ and mortality. ${ }^{5-7}$ Despite improvements in public hygiene and medical care, health disparities across socioeconomic levels have persisted and evenwidened. ${ }^{8}$ Reducing health disparities has risen near the top of the US public health agenda, as reflected in Healthy People 2010.9

In the extensive literature on socioeconomic health disparities, less attention has been paid to examining the variability in health outcomes within social or economic groups. ${ }^{10}$ The approach taken by most studies of SES and health has been to compare health across levels of SES, summarising the health experience within each SES stratum by a single measure, such as mean or rate. This method permits an overall comparison among SES strata, but conceals the variability in outcomes within SES groups. However, understanding the extent of this within group variability in outcomes - and its sources-is an important step in tackling health disparities, for two reasons. The first issue, relevant to public health interventions, is assessing the range of variation in individual vulnerability to the effects of adverse socioeconomic conditions. Because of differences in their genetics, psychosocial circumstances, and other factors, individuals are likely to differ in their susceptibility to the adverse effects of the SES gradient. The interaction of these factors with SES may generate significant differences at both extremes of susceptibility. A resilient subgroup of disadvantaged persons that maintains good health might have important lessons to teach us about those who have successfully weathered disadvantage and the factors that explain their success. Conversely, especially vulnerable subpopulations that suffer disproportionately from social stresses may require special interventions. Thus, by focusing on the heterogeneity within SES groups we can refine our understanding of how individual factors interact with SES to produce population outcomes.

The second reason to study heterogeneity in health outcomes is to further our theoretical models of social causation. An illustrative example of how understanding variability can trigger theoretical insights is provided by research on aging. A robust finding in aging research is that, within the general pattern of age related declines in physical functioning, variability in many physiological parameters increases with age. ${ }^{11}$ To explain those patterns, current theoretical explanations posit that aging results from the accumulation over time of random ("stochastic") damage to structural and biochemical mechanisms for maintenance and repair of physiological systems, eventually resulting in failures of homeostasis. ${ }^{12}{ }^{13}$ The damage derives from both intrinsic and extrinsic factors, but its random progress means that a population of aging people becomes more heterogeneous over time.

In a parallel way, a coherent theory of social causation must account not only for observed social gradients but also for patterns of heterogeneity within those gradients. For example, suppose that within the known association between education and overall health status, less educated groups display less variability in health status than better educated groups. From a psychosocial theory perspective, ${ }^{14}$ perhaps the segregation of low income persons into relatively homogeneous adverse environments translates into exposure to a relatively homogeneous set of psychosocial stressors, leading to relatively homogenous health outcomes. An alternative explanation might posit that the higher mortality rate in low income populations truncates the bottom of their health distributions in population surveys, thereby reducing observable

Abbreviations: SES, socioeconomic status; CTS, community tracking study; PCS, Physical Component Summary; MCS, Mental Component Summary 
heterogeneity. This last hypothesis would also predict that low income populations' diminished variability would become more evident in middle aged and older populations, as the force of differential mortality grew.

On the other hand, suppose the opposite to be true, that less educated groups display more variability in health status. Again, psychosocial theory would postulate that greater variability would emerge from exposure to more heterogeneous patterns of psychosocial stress. Another possibility is that, given a range of individual susceptibility to the effects of socioeconomic position, individual vulnerability functions as an "enabler" of premature illness in unfavourable environments. This last scenario would predict that lower SES would demonstrate a greater effect on lowering the floor of poor health status outcomes (by sickening vulnerable people) than on lowering the ceiling of best possible outcomes (because it is harder to sicken resilient people).

In this study we explore the distribution of a functional health status measure (the Medical Outcomes Study Short Form-12 (SF-12)) both within and across levels of socioeconomic status in order to understand the range of possible outcomes associated with position in the SES spectrum. Is there evidence that certain subpopulations within a given SES stratum are more resilient or vulnerable than others? Is the effect of lower SES on health status to lower the ceiling of best possible outcomes? Or does lower SES pull down the floor of worst possible outcomes? Or both? And what is the net effect on overall variability in health status?

\section{METHODS}

\section{Data source}

This is a secondary analysis of data from the community tracking study (CTS) that was administered to a stratified random sample of the US population between July 1996 and July 1997. ${ }^{15}$ Respondents completed an automated telephone survey; provision was made to sample persons without telephones by providing them with cellular telephones with which to access the survey. Survey questions covered demographics, health insurance, satisfaction with health care, use of health services, and health status. The response rate was $65 \%$. Responses from persons between the ages of 18 and $74(\mathrm{n}=47076)$ were analysed for this study.

The CTS imputed missing data for certain variables using sequential hot-deck imputation methods. ${ }^{16}$ Six per cent of the health status data and about $1 \%$ of the income data used in this study had been imputed. Complete data were available for age.

\section{Measures}

The health status questions administered included the SF-12 questionnaire, ${ }^{17}$ a validated health status instrument with two subscales covering mental and physical health status. The overall SF-12 score and its subscales are all scaled to have a mean of 50 with a standard deviation of 10. The Physical Component Summary (PCS) score and the Mental Component Summary (MCS) subscales are the primary outcomes examined for this study. The PCS is constructed from SF-12 items covering physical limitations, role functioning, pain, and general health status. The MCS is constructed from items on social functioning, role limitations attributable to emotional problems, and mental health (psychological distress and psychological wellbeing.

\section{Analysis}

The survey population was divided into quintiles of family income (cut off values: $<\$ 12000, \$ 12$ 000-25 000, \$25 001$40000, \$ 40001-60000$, and $>\$ 60000$ ) and six age groups (18-24, 25-34, 35-44, 45-54, 55-64, 65-74). For each cell of the age by income matrix, we then calculated mean SF-12

\section{Key points}

- Numerous studies have reported the inverse gradient between socioeconomic position and health.

- Less work has been done to understand the population variability in health outcomes within socioeconomic levels and the extent to which there is resilience or vulnerability to the adverse effects of lower socioeconomic position.

- Lower income groups exhibit more variability in health status than higher income groups. This increased variability stems more from changes in the floor of the health status distribution than from the ceiling. For example, the best $25 \%$ of health status scores in lower income quintiles are virtually identical to the best $25 \%$ of scores in high income quintiles, while the middle and lower segments of the health status distribution are markedly shiffed downwards in lower income persons, beginning in early adulthood.

- Future research should explore the sources and implications of this differential vulnerability to the adverse effects of socioeconomic position.

PCS and MCS scores and the PCS and MCS scores corresponding to the 10th, 25th, 50th, 75th, and 90th centiles of the health status score distribution for that cell. Twenty four of the 30 cells had over 1000 respondents; none had less than 400 respondents. All analyses applied person level weights appropriate to derive estimates for the US population.

We evaluated the variability of physical and mental health status within income strata in several ways. Firstly, we created a series of box and whisker plots of the PCS and MCS centile scores within the age by income cells. The centre line of each box and whisker plot displays the median score; the box's lower and upper ends display the 25th and 75th centiles, respectively; the lower and upper whiskers closest to the box display the 10th and 90th centiles, respectively; and the whisker ends display the extreme values of the distributions (though outliers more than three box lengths from the edge of the box have been trimmed). Secondly, to quantify within group variation, we calculated the 90th-10th centile range, by subtracting the 10th centile score in each cell from the 90th centile score. As a further measure of within group variation, we calculated the standard deviation of the mean within each age by income cell.

We compared the between group variability in physical and mental health status scores among income strata with two approaches. To quantify between group variation by income, we calculated the difference in health status centile scores between the lowest and highest income quintiles. For example, the 90th centile score among 18-24 year olds in the lowest income quintile was subtracted from the 90th centile score in the highest income quintile. The calculation was repeated for the 75 th, 50th, 25 th, and 10th percentiles. Secondly, a Levene's test ${ }^{18}$ for equality of variances was used to evaluate the null hypothesis of equal variances for PCS or MCS scores across income quintiles.

\section{RESULTS}

Table 1 shows the sample sizes by age group and income quintile. The mean SF-12 PCS score for the CTS sample was 50.0 with a standard deviation of 9.9. The mean MCS score was 52.1 with a standard deviation of 9.6. PCS and MCS scores displayed only a weak correlation $(r=0.069$; $\mathrm{p}<0.001$ ). 
Table 1 Sample size (un-weighted) by age category and income group

\begin{tabular}{llllll}
\hline $\begin{array}{l}\text { Income quintile } \\
\text { Age group }\end{array}$ & Low & $\mathbf{2}$ & $\mathbf{3}$ & $\mathbf{4}$ & High \\
\hline $18-24$ & 2278 & 1199 & 687 & 691 & 815 \\
$25-34$ & 2083 & 2408 & 2430 & 1870 & 1301 \\
$35-44$ & 1443 & 1860 & 2504 & 3005 & 3010 \\
$45-54$ & 1111 & 1249 & 1757 & 2226 & 2877 \\
$55-64$ & 1075 & 1089 & 1281 & 1151 & 1223 \\
$65-74$ & 1350 & 1250 & 920 & 533 & 400 \\
\hline
\end{tabular}

Figures $1 \mathrm{~A}$ and $1 \mathrm{~B}$ display box and whisker plots for the PCS and MCS scores by age and income quintile. Several observations can be made from the box plots. Firstly, self reported physical health status was positively related to income and generally declined with age, although there was an upward trend in mean PCS scores in the lowest income quintiles between ages 55-64 and 65-74. Self reported mental health status was also positively related to income, but in contrast with physical health status, generally increased after age 35 .

The second observation is that within group variability in physical and mental health status increases stepwise as income level decreases. A measure of this variability, the range between the 90th and 10th centiles within each age by income group, is displayed in figures $2 \mathrm{~A}$ and $2 \mathrm{~B}$. Variability in both physical and mental health status in middle age (ages 35-44 and 45-54) in the lowest income quintile is twice that in the highest income quintile. Making the same low-high income comparisons using the standard deviation as an alternate yardstick for variability yields differences of $63 \%-$ $75 \%$ in variability in middle age. A Levene's test evaluating the null hypothesis of no statistical differences among the five income specific variances within each age interval was rejected at $\mathrm{p}<0.001$ for both PCS and MCS scores.

The third observation from the box plots is that the greater overall variability in lower income quintiles stems more from variability in the lower centiles (floor) of health status scores than from the upper centiles (ceiling). As elaborated in figures $3 \mathrm{~A}$ and $3 \mathrm{~B}$-which now focus on between group variability-substantial differences in the 10th and 25th centiles of PCS and MCS scores among income quintiles are already apparent at age 18-24 and these changes are magnified over the ensuing three decades. In contrast, the 90th and 75th centiles of PCS and MCS scores show little change among income quintiles. In general, the maximal differences between the lowest and highest quintiles are observed in the 45-54 and 55-64 year old age groups and decrease in the 65-74 year old group.

\section{DISCUSSION}

Considerable variability in age specific health status is evident within SES groups. Although health status is strongly related to socioeconomic status, a subgroup of lower income persons of all ages demonstrates high health status scores. The overall pattern displays a progressive elasticity of the health status distribution with respect to income: the largest effect of lower socioeconomic position is in lowering the floor of worst possible outcomes, while having an intermediate effect on the middle of the distribution, and only minimal effect on lowering the ceiling of best possible outcomes. The effect on the floor of the distribution is large (the 10th centile of PCS or MCS in the lowest income group being 7-20 points lower than the highest income group), impairing physical health status to a degree greater than that observed with major physical illness. For example, in the medical outcomes study, the PCS score averaged 8.67 points lower in those with serious physical illness than in those with minor medical illness only. ${ }^{17}$

The degree of variability in health status is itself strongly related to SES; at each lower level of income, variability progressively increases. In middle aged persons the range of health status encompassed by the 90th and 10th centiles in the poorest quintile was twice that in the most affluent quintile. The observed patterns are similar for both physical and mental health status. The income differences in variability peak in middle age.

Correctly interpreting these findings depends on understanding the sources of variability in health outcomes and particularly why that variability might increase with lower SES. We propose that a useful conceptual model might be a "statistical mechanics of health." Statistical mechanics is the branch of physics concerned with deriving the macroscopic properties of physical systems from the aggregate probabilistic behaviour of large numbers of microscopic units such as atoms and molecules. For example, although the motion of any individual gas molecule is unpredictable, the law of large numbers makes the overall distribution of air molecules in a room very predictable. Applied to social epidemiology, statistical mechanics can help us model how population patterns of illness emerge from individual level events. Although the health trajectory of any individual cannot be predicted with certainty, large numbers of persons exposed to gradients of risk-producing social and physical environments generate the observed social gradients in morbidity and mortality. Broad population level forces, social, economic, and cultural, set the parameters of exposure (What kinds of risks? At what frequency and intensity?) to risk producing social and physical environments that determine overall disease prevalence. The contribution of social epidemiology has largely been to describe the relation between these adverse social circumstances (for example, neighbourhood environment, housing, diet, recreation, job control) and health, with individual SES often serving as a proxy for a set of socially stratified life circumstances. ${ }^{19}$

In the statistical mechanics of health, the societal structuring of experience progressively exposes persons at each lower level of SES to a higher probability of adverse circumstances, ${ }^{20}$ which in turn, through neurobiological ${ }^{21} 22$ and other pathways results in premature morbidity and mortality.

But within those macro patterns, there is heterogeneity within levels of SES that needs explanation, and we will consider three possible sources of heterogeneity. The first source possibility is that macro level social structuring could be, by itself, the major determinant of within SES group variability in health outcomes. Within any SES stratum, significant differences in the political, social, cultural, and economic characteristics of specific areas will produce heterogeneity in outcomes. A key question, however, given the empirical results of this study, is whether there is reason to expect that heterogeneity in social environments should increase as SES decreases. In fact, there is reason to expect the opposite. Greater affluence offers choices to live up or 

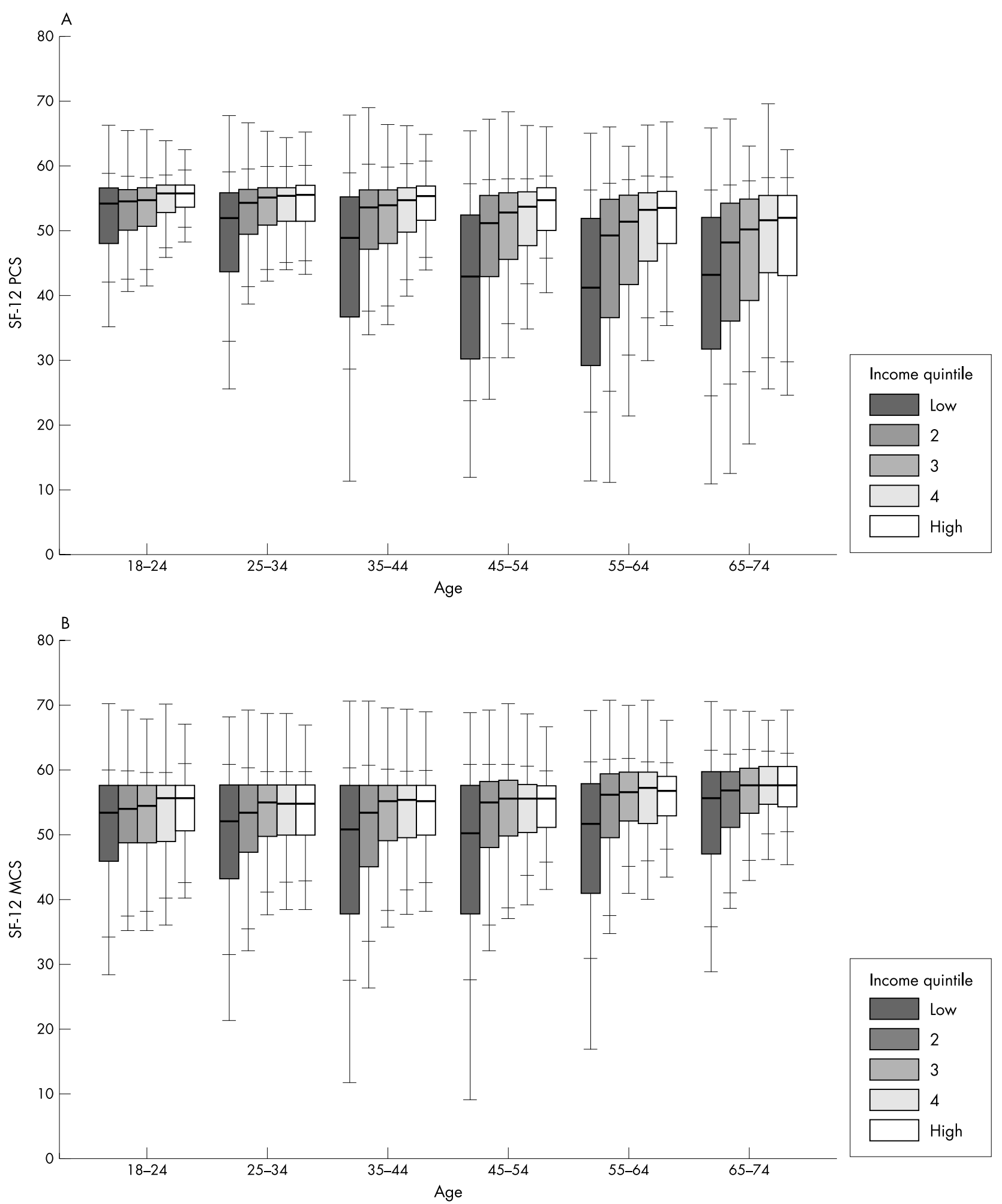

Figure 1 (A) Physical Component Summary box and whisker plots by age and income quintile. (B) Mental Component Summary box and whisker plots by age and income quintile.

down the scale of living environments, depending on how people choose to spend their money, whereas lower income generally can buy entry into only a limited set of living environments. The source of heterogeneity at lower income levels is therefore more probably attributable other factors.

The second candidate source of variability is individual characteristics. Unlike gas molecules, individuals obviously differ in many important respects, and the variable health trajectory of any individual at a given level of SES may arise from individual differences in genetics, ${ }^{23}{ }^{24}$ childhood development, social support, ${ }^{25}$ and psychological traits such as sense of control. ${ }^{26}$ (The distinction between macro and individual factors is analogous to Geoffrey Rose's distinction between the determinants of prevalence and the determinants of 

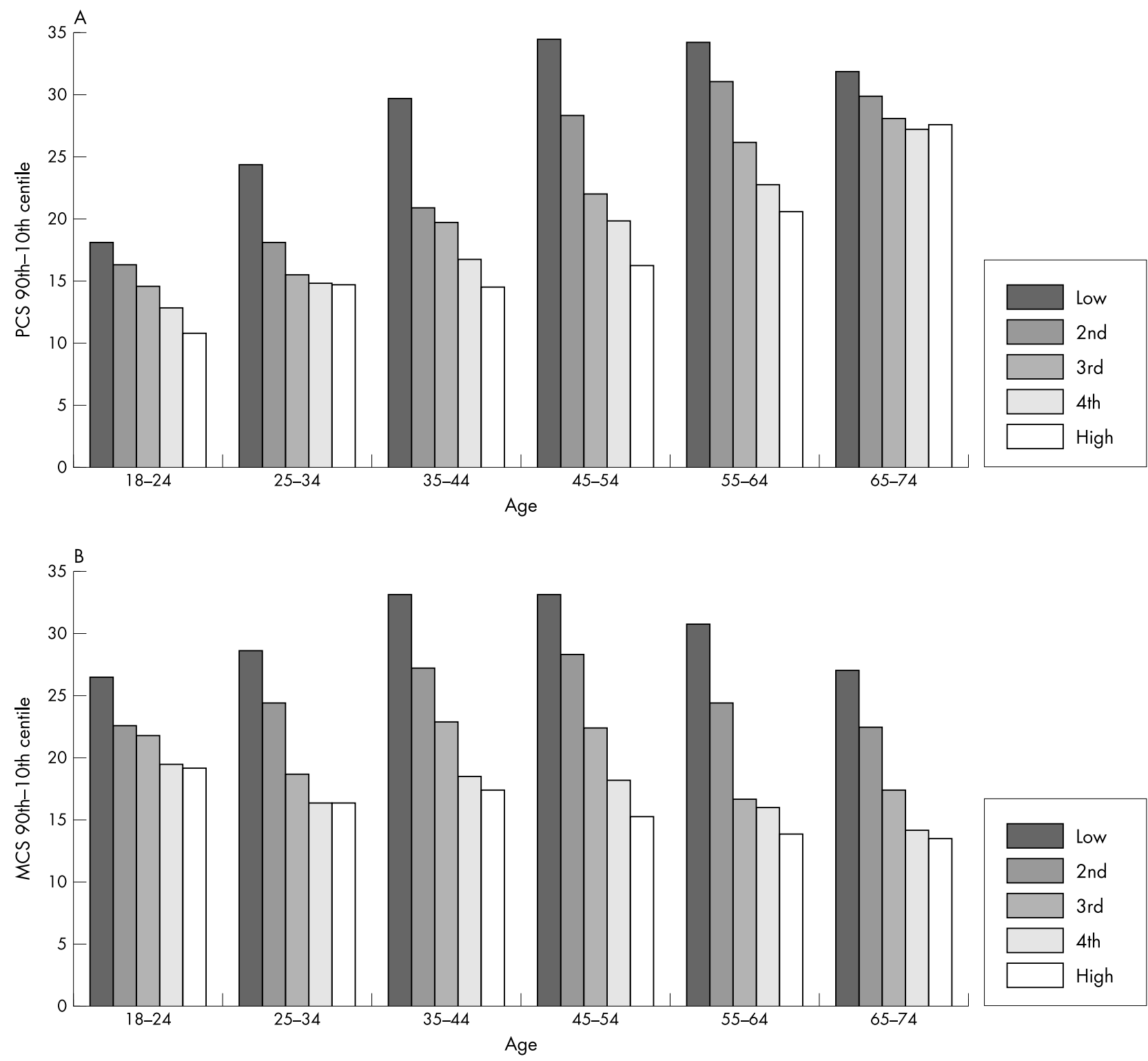

Figure 2 (A) Differences between the 90th and 10th centiles of Physical Component Summary scores by age and income quintile. (B) Differences between the 90 th and 10th centiles of Mental Component Summary scores by age and income quintile.

incidence ${ }^{27}$ ). These individual susceptibility factors can either buffer or exacerbate the effect of socioeconomic position, and we hypothesise that the variability in health status outcomes therefore emerges from the interaction between socioeconomic standing and these individual level factors. Socioeconomic position will have greater effect on health outcomes in susceptible persons.

Presupposing that individual susceptibility factors are evenly distributed by SES (as seems to be the case for genetics, ${ }^{28}$ though highly structured caste societies may prove an exception; see Bamshad et $a l^{29}$ ), the interaction between SES and individual characteristics would be linear; if, however, some individual susceptibility factors are also socially structured into a gradient, as other studies demonstrate, ${ }^{130}$ then a non-linear, multiplicative effect of SES on health would be evident. The social epidemiology literature suggests such a non-linear effect.

The third potential source of increased individual level variability in health within lower socioeconomic groups is a direct physiological effect suggested by the literature on aging. Current theories of aging view it as process without a genetically determined blueprint (because aging occurs after reproduction and is therefore not subject to selection factors for reproductive success), but rather attributable to the cumulative effects over time of randomly occurring damage to structural and biochemical maintenance systems. ${ }^{12}{ }^{13}$ As these maintenance systems fail, the organism becomes susceptible to a broad range of physiological insults from either environmental or internal sources. The observable signature of failing homeostasis is the robust observation that variability in many physiological parameters increases with age. ${ }^{11}$

The parallels between susceptibility observed in aging persons and those with lower socioeconomic status are provocative, including vulnerability to a wide range of pathophysiologically distinct morbidities and the way in which lower SES groups tend to resemble higher SES groups a decade or so older on many health indicators. ${ }^{31}$ A possible mechanism linking adverse social circumstances and morbidity is that lower SES persons' higher rate of exposure to environmental and physiological stresses accelerates and/or exploits the cumulative stochastic damage of aging. 

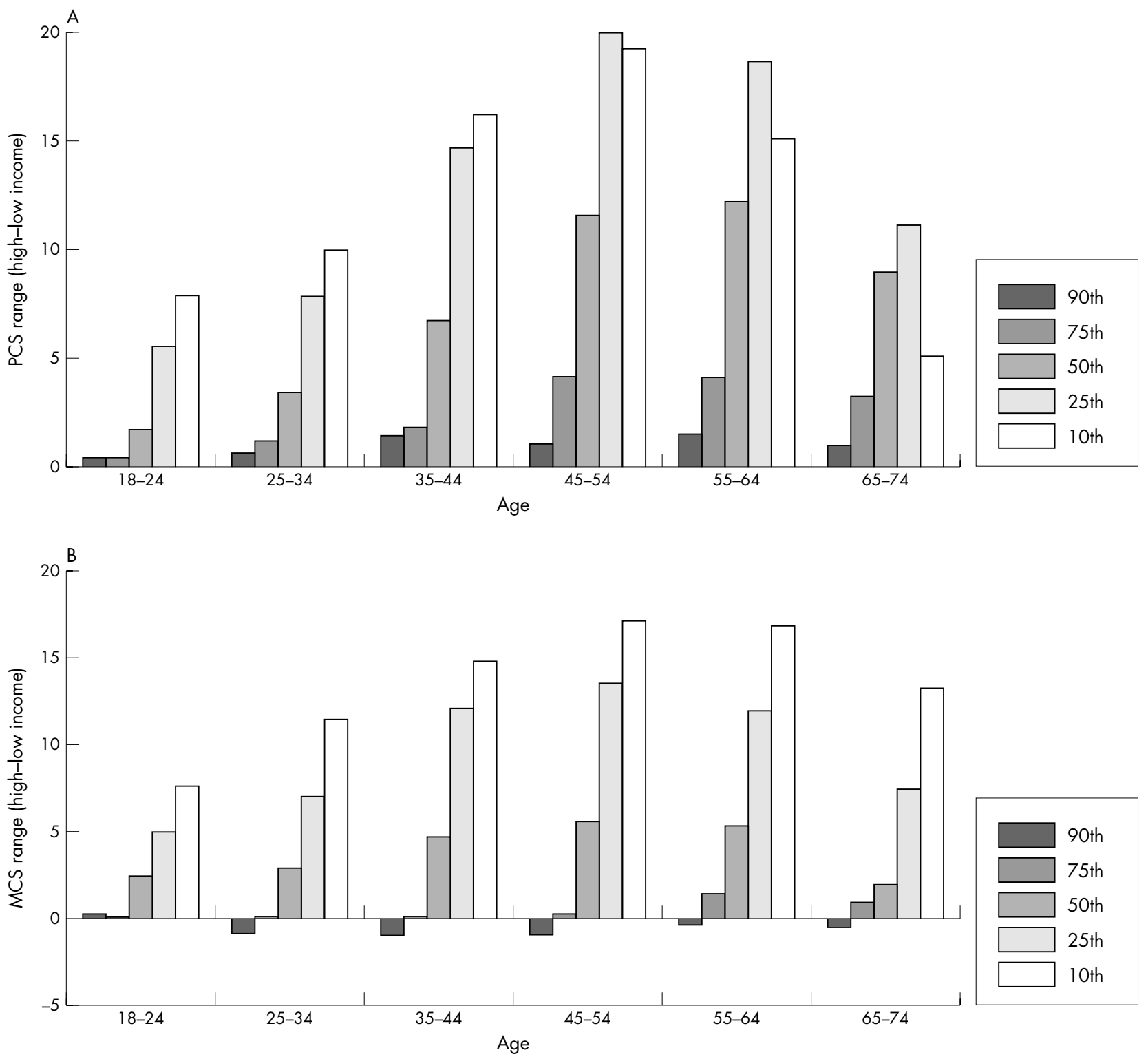

Figure 3 (A) Differences between the lowest and highest income quintiles in the 10th, 25th, 50th, 75th, and 90th centiles of the Physical Component Summary scores, by age. (B) Differences between the lowest and highest income quintiles in the 10th, 25th, 50th, 75th, and 90th centiles of the Mental Component Summary scores, by age.

The strengths of the study are that we analyse a large, nationally representative sample of the US population, apply a well validated measure of health status, the SF-12, and examine a phenomenon that is usually ignored in the health disparities literature-the variability in health outcomes within socioeconomic strata.

At least five potential limitations apply to these results. Firstly, we applied a cross sectional design to the inherently longitudinal problem of age related declines in health status. Although recent data demonstrate that within person changes in population health status measured by the SF-36 are greater than those calculated from cross sectional data, ${ }^{32}$ we cannot verify from our data that individuals track consistently at a given centile of the health status distribution. In fact, we hypothesise that a degree of artefact from the cross sectional design is observable as the increase in physical health status between ages 55-64 and 65-74 in the lowest income quintile. Higher mortality rates among the less affluent selectively cull out those with worse health status, leaving a healthier low income group available for sampling. This hypothesis could be tested with a longitudinal cohort.

Secondly, we used income as our sole stratifying variable for SES, and income is sensitive to health status, raising the potential for reverse causation, wherein poor health leads to low income rather than vice versa. To assess this, we repeated our analyses using education as the stratifying variable and the patterns were unchanged.

Thirdly, it is possible that ceiling effects on the SF-12 obscured our ability to detect differences in health status at the highest reaches of the health status scale. This concern is lessened by the observation that the 90th centile scores on the SF-12 PCS and MCS were still 10 points below the maximum scores in the sample, but the scales may not adequately differentiate between gradations of excellent health. Fourthly, we examined only the association between SES and self rated health-it is possible that other health outcomes will not demonstrate the same pattern we have described here. Fifthly, respondents' estimates of their 
physical functioning are biased by factors unrelated to their physical health, such as degree of neuroticism. Perhaps those who score high on the SF-12 are predisposed to view life through rose coloured lenses, no matter what their objective level of functioning. ${ }^{33}$

In summary, we find that the best $25 \%$ of health status scores in lower income quintiles are virtually identical to the best $25 \%$ of scores in high income quintiles; it is the middle and lower segments of the health status distribution that are markedly shifted downwards in lower income persons, beginning in early adulthood. There seems to be a resilient subgroup of lower SES people whose self rated health remains excellent throughout life, while vulnerable persons of lower SES see a rapid deterioration in health status as they reach middle age. The findings remind us that within the "deterministic" social structuring of health there are differences in individual resilience and vulnerability that are amenable to further exploration and potential modification. Understanding these individual-environment interactionsthe "ecology" of social causation-will deepen our understanding of social epidemiology.

\section{Authors' affiliations}

R L Ferrer, R Palmer, Department of Family and Community Medicine University of Texas Health Science Center at San Antonio, San Antonio, Texas, USA

\section{REFERENCES}

1 Marmot M, Davey Smith G, Stansfeld S, et al. Health inequalities among British civil servants: the Whitehall II study. Lancet 1991;337:1387-93.

2 Fiscella K. Is lower income associated with greater biopsychosocial morbidity? Implications for physicians working with underserved patients. J Fam Pract 1999;48:372-7.

3 Larson RJ, Kasimatis M. Day-to-day physical symptoms: individual differences in the occurrence, duration, and emotional concomitants of minor daily illness. J Pers 1991;59:387-423.

4 Hemingway $\mathbf{H}$, Nicholson A, Stafford $M$, et al. The impact of socioeconomic status on health functioning as assessed by the SF- 36 questionnaire: the Whitehall II study. Am J Public Health 1997;87:1484-90.

5 Marmot M, Shipley M, Rose G. Inequalities in death: specific explanations of a general pattern? Lancet 1984;i:1003-6.

6 Davey Smith G, Neaton JD, Wentworth D, et al. Socioeconomic differentials in mortality risk among men screened for the Multiple Risk Factor Intervention Trial: I. White men. Am J Public Health 1996;86:486-96.

7 Davey Smith G, Neaton J, Wentworth D, et al. Socioeconomic differentials in mortality risk among men screened for the Multiple Risk Factor Intervention Trial: II. Black men. Am J Public Health 1996;86:497-504.

8 Pappas G, Queen S, Hadden W, et al. The increasing disparity in mortality between socioeconomic groups in the United States, 1960 and 1986. N Engl J Med 1993;329:103-9.
9 US Department of Health and Human Services. Healthy people 2010: understanding and improving health. Washington, DC: US Government Printing Office, 2000.

10 Evans RG, Barer ML, Marmor TR, eds. Why are some people healthy and others not? New York: De Gruyter, 1994.

11 Rowe JW, Kahn RL. Human aging: usual and successful. Science 1987;237: 143-9.

12 Kirkwood TBL, Austad SN. Why do we age? Nature 2000;408:233-8.

13 Rattan SIS. Biogerontology: the next step. Ann NY Acad Sci 2000;908:282-90.

14 Krieger $\mathrm{N}$. Theories for social epidemiology in the 21 st century: an ecosocial perspective. Int J Epidemiol 2001;30:668-77.

15 Center for Studying Health System Change. Community Tracking Study Household Survey, 1996-7: [United States] [Public Use Version] [Computer file]. 3rd ICPSR version. Washington, DC: Center for Studying Health System Change [producer], 1999. Inter-university Consortium for Political and Social Research [distributor], 2000.

16 Center for Studying Health System Change. User guide for public-use version of main data file. 3rd ICPSR version. Washington, DC: Center for Studying Health System Change, February, 2000.

17 Ware JE Jr, Kosinski M, Keller S. A 12-Item Short-Form Health Survey. Med Care 1996;34:220-33.

18 Levene $\mathrm{H}$. Contributions to probability and statistics: essays in honor of Harold Hotelling. In: Olkin I, Ghurye SG, Hoeffding W, et al, eds. Palo Alto: Stanford University Press, 1960:278-92.

19 J W Lynch, G A Kaplan. Socioeconomic position. In: Berkman LF, Kawachi I, eds. Social epidemiology. Oxford: Oxford University Press, 2000:3-35.

20 Pearlin LI. The sociological study of stress. J Health Soc Behav 1989;30:241-56.

21 McEwen BS. Protective and damaging effects of stress mediators. N Engl J Med 1998;338:171-9.

22 Baum A, Garofalo JP, Yali AM. Socioeconomic status and chronic stress: does stress account for SES effects on health? In: Adler N, Marmot M, McEwen BS, et al. Socioeconomic status and health in industrial nations. Ann NY Acad Sci 1999:131-144.

23 Caspi A, McClay J, Moffitt TE, et al. Role of genotype in the cycle of violence in maltreated children. Science 2002;297:851-4.

24 Lynch JW, Everson SA, Kaplan GA, et al. Does low socioeconomic status potentiate the effects of heightened cardiovascular responses to stress on the progression of carotid atherosclerosis? Am J Public Health 1998;88:389-94.

25 House JS, Landis KR, Umberson D. Social relationships and health. Science 1988;241:540-5.

26 Bosma $\mathrm{H}$, Marmot $\mathrm{M}$, Hemingway $\mathrm{H}$, et al. Low job control and risk of coronary heart disease. BMJ 1997;314:558-65.

27 Rose G. Sick individuals and sick populations. Int J Epidemiol 1985;14:32-8.

28 Holtzman NA. Genetics and social class. J Epidemiol Community Health 2002;56:529-35.

29 Bamshad MJ, Watkins WS, Dixon ME, et al. Female gene flow stratifies Hindu castes. Nature 1998;395:651-2.

30 Taylor SE, Repetti RL. Health psychology: what is an unhealthy environment and how does it get under the skin? Annu Rev Psychol 1997:48:411-47.

31 Hayward MD, Crimmins EM, Miles TP, et al. The significance of socioeconomic status in explaining racial gaps in chronic health condititions. Am Sociol Rev 2000;65:910-30.

32 Hemingway $H$, Stafford $M$, Stansfeld S, et al. Is the SF-36 a valid measure of change in population health? Results from the Whitehall II study. BMJ 1997;315:1273-9.

33 Muldoon MF, Barger SD, Flory JD, et al. What are quality of life measures measuring? BMJ 1998;316:542-5. 